\title{
Chronic Fatigue Syndrome: Where is The Reality?
}

\author{
Adel Mahmoud AE \\ Department of Internal Medicine, Ain Shams University; Egypt.
}

\begin{abstract}
A lot of patients present with complaints of postexertional malaise, persistent flulike symptoms, unrefreshing sleep, "brain fog," and often a long list of other symptoms. Some appear ill, but many don't and the routine laboratory tests often come back negative. these are the known manifestations of chronic fatigue syndrome (CFS). Unfortunately, those patients are frequently labeled as malingerers, depressed, or at least partially psychosomatic.

It affects about 1 million adults and children in the United States. Women outnumber men in CFS diagnosis, although about a quarter are males. The condition can appear at any age and appears to be more common among ethnic and racial minority groups and in those of lower socioeconomic status. ${ }^{1}$

Cognitive dysfunction and chronic pain are common manifestations. Other frequent symptoms include orthostatic intolerance "particularly a postural orthostatic tachycardia syndrome" which can be elicited by a tilt-table test, gastrointestinal dysfunction including irritable bowel syndrome, heat or cold intolerance, and persistent flulike symptoms.

Many patients also meet criteria for fibromyalgia. In addition to fibromyalgia, other common co-morbid conditions include irritable bowel syndrome, joint hypermobility, interstitial cystitis, and migraines.
\end{abstract}

The name of CFS has been used in the United States since 1988; in the United Kingdom, Canada, and elsewhere it is called myalgic encephalomyelitis (ME).

Experts conceptualize ME/CFS as an abnormal immune system response to a number of infectious or environmental triggers and stressors, resulting in a chronic state of inflammation, autonomic dysfunction, impaired hypothalamic-pituitary-adrenal axis, and neuroendocrine dysregulation. But It is still unknown whether it's autoimmune or chronic low-grade infection.

On the other hand, there are many controversies about CFS, is it a real disease. The name itself is one of the many controversies surrounding the condition and how can we diagnose disease without a diagnostic test, classification criteria, known pathogenesis, or a proven treatment ?. "Obviously, the first thing we have to do is to see that is it a real disorder?.

For Correspondence: adel.mahmoud@med.asu.edu.eg
There are now abundant evidences that in these patients there is an underlying biological process.

CFS as defined in 1994 by the Centers for Disease Control and Prevention (CDC) represents a more heterogeneous and less severely affected population than does ME captured by the 2003 Canadian Clinical Case Definition. Both definitions require multiple symptoms in addition to 6 months of unexplained fatigue to make the diagnosis, but the $\mathrm{ME}$ criteria also require the hallmark symptom of postexertional malaise. $^{2}$

\section{Evidence of a Real Disease:}

Such evidence includes significantly reduced oxygen consumption and workload for ME/CFS patients after treadmill tests ${ }^{3}$, and altered gene expression compared with controls following moderate exercise. ${ }^{4}$

Other biological evidence includes a recent finding of bilateral white matter atrophy in ME/CFS patients compared with controls ${ }^{5}$, several studies documenting significant decreases in natural killer cell cytotoxic activity, and increased levels of multiple pro-inflammatory cytokines. ${ }^{6}$

A highly significant elevation in incidence of non-Hodgkin lymphoma "which, like ME/CFS, has been linked to Epstein-Barr virus" ${ }^{7}$ was found among ME/CFS patients aged 66-99. In a novel study of 165 consecutive patients with ME/CFS who underwent upper gastrointestinal endoscopies and antrum biopsies, 135/165 (82\%) stained positive for enterovirus viral capsid protein 1 compared with just $7 / 34(20 \%)$ of controls $(\mathrm{P} \leq .001){ }^{8}$

In another novel finding, approximately $2 \%$ of $\mathrm{ME} / \mathrm{CFS}$ cases were found to have chromosomally integrated human herpesvirus-6 (HHV-6) as compared to just $0.2 \%-0.85 \%$ of the general population, ${ }^{9}$ suggesting a specific etiology for a small proportion of cases. $^{10}$

Responses to treatment in randomized, blinded, placebo-controlled trials also point to biological causation, including improvements with valganciclovir in a study of ME/CFS patients with elevated antibody titers to HHV-6 and Epstein-Barr virus $^{11}$, and a preliminary trial (now being repeated in a larger patient group) in which ME/CFS patients responded to rituximab, a monoclonal antibody that targets B cells and is used for the treatment of non- 
Hodgkin lymphoma and other B-cell-mediated conditions. $^{12}$

The investigational immuno-modulatory doublestranded RNA drug rintatolimod (Ampligen ${ }^{\circledR}$; Hemispherx Biopharma, Inc.) produced objective improvement in exercise tolerance and other endpoints in a phase 3 prospective, double-blind, randomized, placebo-controlled trial of 234 subjects with longstanding, debilitating ME/CFS. ${ }^{13}$

There are several pro-inflammatory cytokines identified that are significantly elevated in samples from $200 \mathrm{ME} / \mathrm{CFS}$ patients compared with 400 ageand gender-matched controls, and that correlate with illness severity. It's an indicator that what you are seeing is real.

It is believed that, in ME/CFS "there is a genetic predisposition for an overwhelming inflammatory response to an infectious agent that was supposed to help the patient but is overwhelming, triggering a tremendous inflammatory cascade." This model suggests a possible role for anti-inflammatory medications as treatment, noting that both the valganciclovir and rituximab studies suggest proof of concept for that approach.

Many of the infectious agents that have been linked to ME/CFS, "including Epstein-Barr virus ${ }^{14}$, HHV-6 ${ }^{15}$, Coxiella burnetii ("Q fever") ${ }^{16}$, Ross River virus in Australia ${ }^{17}$, and various enteroviruses" ${ }^{18}$ are ones that can't be fully eradicated by the immune system even in healthy people and/or are capable of infecting the central nervous system. This suggests the possibility that some ME/CFS patients may have a chronic, low-level encephalitis.

Still, these biomarker data doesn't add up to an easy diagnostic test. Moreover, there are many difficulties with these biomarker studies, as small study sample sizes, the wide heterogeneity of the criteria used for patient selection leading to inconsistent findings, and the overlap of abnormalities found in ME/CFS patients with those of other conditions. Furthermore, technologies used in some of the studies, such as functional MRI and cytometry, are too expensive and impractical to be employed routinely in clinical settings.

"Based on the current state of research on this topic, biomarkers offer a strong potential for characterizing [ME/CFS] subgroups in terms of clinical phenotypes, endophenotypes, prognosis, and response to therapy."

\section{REFERENGES}

1. Steele L, et al. Am J Med. 1998;105:83S-90S.

2. Jason LA, et al. Fatigue. 2013;1:168-183.

3. Snell CR, et al. Phys Ther. 2013;93:1484-1492.

4. $\quad$ Light AR, et al. J Pain. 2009;10:1099-1112.

5. Zeineh MM, et al. Radiology. 2014.

6. Klimas NG, et al. Brain Behav Immun. 2012;8:1202-1210.

7. Coghill AE, \& Hildesheim A. Am J Epidemiol. 2014;180:687-695

8. Chia JK, and Chia AY. J Clin Pathol. 2008;61:43-48.

9. Pantry SN, et al. J Med Virol. 2013;85:19401946.

10. Lum E, et al. Future Microbiol. 2014;9:433-436.

11. Montoya JG, et al. J Med Virol. 2013;85:21012109.

12. Fluge $\varnothing$, et al.. PLoS One. 2011;6:e26358.

13. Strayer DR, et al; PLoS One. 2012;7:e31334.

14. Loebel M, et al. PLoS One. 2014;9:e85387.

15. Komaroff AL. J Clin Virol. 2006;37 Suppl 1:S39-S46.

16. Limonard GJ, et al. QJM. 2010;103:953-958.

17. Selden SM, and Cameron AS. Med J Aust. 1996; 165:313-317.

18. Chia J, et al. J Clin Pathol. 2010; 63: 165-168 